\title{
DIOS-AMOR Y FILOSOFÍA DE LA EDUCACIÓN \\ DIÁLOGO ABIERTO CON ALGUNAS DISCUSIONES \\ PEDAGÓGICAS ACTUALES DESDE UNA OLVIDADA \\ FILOSOFÍA CRISTIANA DEL AMOR
}

\author{
GOD-LOVE AND PHILOSOPHY OF EDUCATION \\ OPEN DIALOGUE WITH SOME CURRENT PEDAGOGICAL DISCUSSIONS \\ FROM A FORGOTTEN CHRISTIAN PHILOSOPHY OF LOVE
}

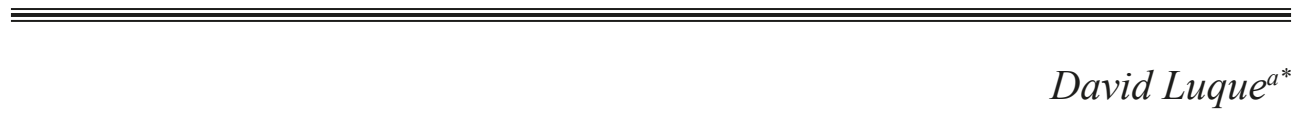

Fechas de recepción y aceptación: 26 de febrero de 2021 y 25 de marzo de 2021

DOI: https://doi.org/10.46583/edetania_2021.59.801

Resumen: El objetivo de este artículo es analizar las aportaciones que una filosofía cristiana del amor puede hacer a las discusiones pedagógicas sobre el amor que se han desarrollado en el siglo XXI. Para ello, se estructura en dos partes con dos objetivos diferenciados. En la primera se estudian una serie de obras sobre el amor producidas en el siglo xx por intelectuales cristianos para abstraer algunos elementos comunes a todas ellas que permitan hablar de rasgos básicos. En la segunda, después de una revisión sistemática de las principales discusiones desarrolladas en el ámbito de la filosofía educativa, se aportan posibles líneas de pensamiento desde los elementos básicos de una filosofía cristiana del amor. A la postre, se aporta una voz cristiana renovada a las discusiones actuales sobre el amor en filosofía de la educación.

Palabras clave: filosofía de la educación, cristianismo, tendencias educativas, disciplinas intelectuales, relación profesor-estudiante.

Abstract: The main aim of this article is to analyze the contributions that a Christian philosophy of love can make to the pedagogical discussions about love developed in the $21^{\text {st }}$ century. For this purpose, the article is structured in two parts with two different objectives.

a Departamento de Ciencias de la Educación, Lenguaje, Cultura y Artes. Ciencias Histórico-Jurídicas y Humanísticas y Lenguas Modernas. Universidad Rey Juan Carlos.

${ }^{*}$ Correspondencia: Universidad Rey Juan Carlos. Facultad de CC. Jurídicas y Sociales. Camino del Molino, 5. 28942 Fuenlabrada (Madrid), España.

E-mail: david.luque@urjc.es 
In the first part, a series of philosophical works on love produced in the twentieth century by Christian intellectuals are studied to abstract some elements common to all of them which will allow us to speak of basic features. In the second part, after a systematic review of the main discussions on love developed in the field of educational philosophy, possible lines of thought are provided from the basic elements of the previous section. In the end, a renewed Christian voice is introduced to current discussions of love in the philosophy of education.

Keywords: educational philosophy, Christianity, educational trends, intellectual disciplines, teacher-student relationship.

Es una mano intentando coger del amor algún pedazo.

Kutxi Romero

\section{INTRODUCCIÓN}

Todavía se olía la pólvora quemada que asoló el continente europeo durante las dos grandes guerras cuando los intelectuales cristianos comenzaron a preguntarse sobre la imagen de sí mismo que Dios había revelado entonces. Los teólogos situaron la respuesta en la comprensión de una imagen renovada de Dios-Trinidad. Los filósofos cristianos creyeron que era necesaria una comprensión más profunda y actualizada del amor. Aunque ambos parecieron entender que la unidad y la fraternidad eran las respuestas que la humanidad debía dar al desastre de las guerras, la shoah y los gulags, corrieron suertes dispares en la historia del pensamiento. La corriente teológica se desarrolló hasta el punto de permear el magisterio aún hoy, mientras que la filosófica cayó en el olvido casi por completo. Así las cosas, este artículo desea recuperar algunos de los pensamientos sobre el amor cristiano más sustanciales que se produjeron en esa fugaz e intensa eclosión filosófica sobre el amor a través de los textos de Dietrich Von Hildebrand, Gustave Thibon, Hans Urs Von Balthasar, Søren Kierkegaard, C. S. Lewis, Vladimir Soloviev y Lev Tolstoi.

Pero quiere situar sus aportaciones en el terreno pedagógico y, por ello, aspira también a establecer un diálogo con las discusiones educativas actuales que estudian el amor. Porque en la filosofía de la educación, el amor ha constituido una constante discursiva que sigue viva hoy, cuando parece haberse revelado como un núcleo de resistencia frente a la cosificación de los sistemas 
educativos y la corrosión de la identidad docente. Sin embargo, esos mismos artículos revelan un vacío elocuente sobre las aportaciones del cristianismo o transmiten interpretaciones superficiales y hasta erróneas: sitúan el amor cristiano al margen del eros o la philia, desarrollan exégesis que no están basadas en los textos originales, se refieren siempre y casi en exclusiva a Pablo, San Agustín o Santo Tomás, o dibujan el amor cristiano como una renuncia a la propia felicidad que la hace indeseable para la vida de las aulas.

Así las cosas, este artículo pretende analizar las aportaciones que la filosofía cristiana del amor desarrollada en el siglo xx puede hacer a las discusiones pedagógicas sobre el amor que se han desarrollado en la actualidad. Para ello se estructura en dos partes. En la primera, se profundiza en esas aportaciones sustanciales sobre el amor cristiano y se intenta abstraer una serie de elementos comunes. En la segunda, después de una revisión sistemática de las principales discusiones, se aportan posibles líneas de pensamiento desde los elementos básicos de una filosofía cristiana del amor. A la postre, se rescata ese episodio luminoso de la reflexión cristiana para aportar una voz renovada a las discusiones actuales. Con todo, me parece necesario un pequeño matiz antes de comenzar. El énfasis que se hace aquí en el amor obedece a que constituye su objeto de estudio, pero no desea incurrir en el siguiente error: el amor no es Dios, porque eso sería casi una idolatría, sino que Dios es amor; Dios constituye el único punto de partida que cabe asentar aquí. La única confesión de fe válida.

\section{Primera parte. Una olvidada filosofía del AMOR CRISTIANO EN EL SIGLO XX. AproXimación ANTOLÓGICA Y SISTEMÁtiCA}

¿Qué criterios se han seguido para seleccionar a los autores? Que todos publicaron obras filosóficas que estudiaban específicamente el amor cristiano en el siglo xx, a las que habría que añadir dos excepciones. Kierkegaard, que escribió previamente, y Balthasar, cuya obra es de naturaleza teológica ${ }^{1}$. Sobre

\footnotetext{
${ }^{1}$ Habrá a quien le pueda parecer extraño que, admitiendo como excepción una obra de naturaleza teológica, esta haya sido la de Balthasar y no la de otros autores con, quizá, mayor repercusión como Amor y responsabilidad, que escribió san Juan Pablo II antes de acceder al pontificado, o Deus Caritas Est, que es la encíclica con la que Benedicto XVI abrió su magisterio. El criterio que he seguido para
} 
esa base, se desarrollan dos apartados. Uno estudia las obras de cada autor para comprender la estructura básica de su pensamiento. Otro condensa los elementos básicos del amor cristiano que concitan la unanimidad de todos los autores y que sirven de base para establecer el diálogo con la pedagogía que vendrá en la segunda parte del texto.

\subsection{Una pequeña historia del amor cristiano. Antología y síntesis}

El catolicismo aporta el pensamiento de Hildebrand, Thibon, Soloviev y Balthasar.

El amor en Hildebrand, que publicó en La esencia del amor de 1971, es la respuesta dada al valor que se ve en el objeto amado, que aparece como lo más digno de amor por sus cualidades (1998, pp. 49-57). Esta idea del amor se movería entre la intentio unionis e intentio benevolentiae. En una unión entre los amantes que encuentra su cauce de expresión en una entrega al amado que no significa la pérdida de las cualidades individuales, sino que, si es recíproca -y debe serlo-, genera una unidad espiritual hasta tal punto que cuanto sucede al otro se vive como propio (1998, p. 189). En el ámbito de la caridad es preciso hablar del amor a Dios y el amor al prójimo². Aquí es necesario observar tres ideas. Que el MN se funda en el amor de Dios y nace a partir de él, lo que provoca que su obediencia nazca de la insinuación de la propia voz de Cristo (1998, p. 297). Que la intención unitiva y la benevolente no es una entrega o una interiorización de lo que sucede al amado, sino una actualización de la bondad en el alma del amante (1998, pp. 295-296). Que la caridad inunda todas las categorías del amor natural (1998, pp. 298-302). Escribe Hildebrand: "La palabra específica del amor, que es pronunciada en cada una de sus formas, solo puede alcanzar un desarrollo puro y pleno si son irrigadas por la caridad"

no incluirlas en esta excepción es doble. El primero, que no había lugar para estudiarlas en el corto espacio de un artículo si quería a otros autores más olvidados. El segundo, que son obras que proceden de personas que llegaron a ser pontífices de la Iglesia católica y, en ese sentido, han sido textos ya muy estudiados también para la pedagogía.

${ }^{2} \mathrm{MN}$, en adelante. MN: Mandamiento Nuevo, que se refiere al mandato de "que os améis unos a otros; como yo os he amado, amaos también unos a otros" (Jn 13, 34). 
(1998, p. 306). Así, la conciencia moral puede expresarse a través de cada uno de ellos (1998, p. 319).

Thibon, el francés nominado cuatro veces al Nobel que escribió Sobre el amor humano en 1961, lo entendió como una unión que se alcanza tras integrar ascéticamente la división entre lo que él llama "vida" y "espíritu" (2010, pp. 14-15) y que habría sido originada por la caída original y agravada por la responsabilidad humana (2010, pp. 18-19) que continuamente buscaría ídolos que le alejan de Dios (2010, p. 28). Así, cuando el espíritu logra conducir la vida (2010, p. 20), aparece un amor que se comprende en función de cuatro aspectos. El primero, que el amor tiende a romper el aislamiento del hombre provocado por su idolatría para abrirlo a la unidad con el otro/Otro, que es también su yo más profundo (2010, p. 37). El segundo, que el amor irriga toda la naturaleza humana valiéndose así de los instintos y de la carne para amar en una nueva unidad (2010, p. 125). El tercero, que los amores naturales evolucionan hacia la perfección cuando los componentes humanos y sensibles concuerdan con el amor divino (2010, p. 86). Finalmente, se puede hablar de una transfiguración del amor en donde el ser humano aprende a amar a las personas como fines en sí mismos, es decir, por causa de su pobreza y sus imperfecciones (2010, pp. 138-139). En suma, la transfiguración auténtica sucede cuando se ama a Dios a través del amado (2010, pp. 148-150).

En El significado del amor, Soloviev subraya su dimensión unitiva. En el ruso, el amor ha de comprenderse como una dinámica que se articula en dos movimientos. Un movimiento de individualidad, en que la persona vence su egoísmo y es afirmada y afirma a las otras en su valor absoluto (2009, p. 71), y un movimiento de unión, en que dos personas - y más progresivamente-se unen en la aspiración a la unitotalidad (2009, p. 107). Así, el amor da valor absoluto al objeto amado a través de la unión con él no creando una realidad nueva, sino llevando a su máxima plenitud lo que se encontraba de forma embrionaria en ambas partes, que es esa pretensión de unidad (2009, pp. 71-72). De esta manera se puede restaurar la imagen de Dios en la humanidad-Soloviev dirá que incluso físicamente-: porque el amor manifiesta la imagen de Dios en el hombre como transfigurándolo cuando ama (2009, pp. 72-73). En suma, “el hombre particular puede salvarse realmente, puede regenerar y perpetuar la propia vida individual en el amor auténtico solo si vive en comunión y junto con todos" (2009, p. 111). Sobre esta base, termina fundamentando la conciencia. 
El texto que Balthasar escribió en 1963, Solo el amor es digno de fe, comienza preguntándose sobre la posibilidad de conocer el amor. Esto es posible porque el amor que Dios tiene al mundo es reconocible incluso aunque él sea totalmente Otro (2004, p. 77), dado que es incoado en las personas un amor sobrenatural $(2004$, pp. 60, 77) que hace que no se pueda conocer de forma gnóstica (2004, pp. 107-108). De esta manera, hay en el ser humano un germen de amor que es imago dei (2004, p. 79) y que se manifiesta externamente en correspondencia a una revelación que es el amor (2004, p. 85) manifestado trinitariamente en el hecho objetivo de la cruz (2004, pp. 88-91, 101-102, 107-108). Pero ¿cómo puede amar el ser humano en su respuesta libre a la cruz? Hay que comenzar diciendo que el amor no tiene forma creatural, sino que, a lo sumo, adopta la forma de un "sí" que es siempre una respuesta a la primera iniciativa de Dios (2004, pp. 123-124). Entonces, el amor de Dios penetra en la libertad del ser humano haciéndolo cada vez más amor, como una regeneración original al amor donado a la persona (2004, p. 106). Así, “el amor lo posee a él [al ser humano] en lo más íntimo, interius intimo meo. El amor organiza al hombre y no a la inversa" (2004, p. 132). De esta manera, las virtudes naturales quedan transfiguradas por el amor y "los valores supremos de la ética individual se caen sin el amor: únicamente el amor lleva a plenitud toda ley en él compendiada" (2004, p. 128). Y es así como cobra sentido un obrar cristiano dirigido interiormente por el amor de Dios, que toma la forma de la libertad humana. El MN no es sino impedir la competencia de otros objetos que pueden erigirse en ídolos (2004, pp. 109-110) así como todos los presupuestos anteriores se dirigen al encuentro con el prójimo (2004, pp. 112-116). Todo lo cual culmina con la reciprocidad del amor, que parece que es exclusivo de un amor trinitario, donde "el amor no quiere más recompensa que el amor que corresponde" (2004, pp. 109, 118).

Kierkegaard y Lewis representarían otras confesiones cristianas.

Las obras del amor, que Kierkegaard publicó en 1847, ofrecen tres intuiciones fundamentales. La primera es que el amor permanecería oculto en el interior del ser humano, nutriéndose del amor de Dios como un manantial se nutre de un afluente, y solo es posible conocerlo a través de las obras realizadas por las personas (2006, pp. 25-26). Una de esas manifestaciones es la obediencia a la ley regia, al MN, que nace del recto amor a uno mismo y vuelve prójimos entre sí a todos los seres humanos (2006, pp. 44, 82). Esta obligatoriedad de amar 
eterniza al amor y le da entidad ontológica (2006, pp. 52-53) a la vez que libera al ser humano de la dependencia del amado porque el amor ya no depende del objeto amado, sino del puro mandato (2006, p. 61). "El amor auténtico, el amor que, convirtiéndose en deber, sufrió el cambio de la eternidad, no varía jamás" (2006, p. 55), escribe Kierkegaard. Así las cosas, el amor se vuelve un asunto de conciencia moral. Pues si el amor fluye en el ser humano como la sangre en las venas (2006, p. 169), entonces, las relaciones entre los seres humanos son relaciones de amor donde la conciencia pasa también a ser amor (2006, p. 169). De esta manera, el cristianismo irrumpe en el discurso sobre el amor no para enseñar a amar de un modo determinado, sino para revelar cómo amar de un modo general a todo ser humano y por eso transforma internamente, según el espíritu, todos los otros tipos de amores (2006, pp. 177-181).

En 1960, Lewis publicó Los cuatro amores, donde propuso una comprensión de los amores naturales en la intersección de dos ejes. El eje compuesto por el "amor dádiva", que es un amor gratuito que se dona, y el "amor necesidad", que surge de la pura necesidad del ser humano (2017, pp. 11-12). Y el eje de los "placeres necesidad", que son aquellos que mueren en nosotros cuando los hemos alcanzado, y los "placeres de apreciación", que no han exigido que se aprecien valorativamente (2017, pp. 26-27). Pero su aportación fundamental para este artículo es la idea de que todos los amores naturales nacen del amor de Dios. Escribe: "los amores naturales no son auto-suficientes. [...] Decir esto no es empequeñecer los amores naturales, sino indicar dónde reside su verdadera grandeza" (2017, p. 155). Esta dependencia provoca tres efectos. El primero que, aceptando su carácter secundario respecto del amor de Dios, los amores naturales se liberan y se desarrollan hacia una mayor plenitud (2017, p. 158). El segundo es que Dios "implanta en nosotros todos los amores-dádiva como los amores-necesidad" (2017, p. 169) y, sobre esa base, podemos amar a Dios mismo adorándole, y amar lo que no es digno de amar y ser amados como indignos en el cumplimiento del MN (2017, p. 170). El tercero es una interpretación escatológica del amor, pues los amores naturales quedarían vivificados por el amor divino hasta tal punto que, al ver a Dios cara a cara, se experimentará la sensación de haberlo visto ya en cada acto de amor natural transfigurado (2017, p. 184). Dice Lewis que la caridad "no se rebaja haciéndose simple amor natural, sino que el amor natural es asumido -haciéndose su instrumento obediente y armónico- por el Amor en sí mismo” (2017, p. 177). 
Tolstoi, que fue bautizado en la Iglesia ortodoxa y se mostró siempre muy crítico con todas las formas eclesiales, aporta un pequeño texto.

La idea sobre la que construye su comprensión del amor en 1908 es que la humanidad vive orientada por la "ley de la violencia", que somete a los hombres bajo la guía de un régimen que les oculta el verdadero mensaje del evangelio (2018, pp. 25-30), que sería la "ley del amor". La caridad, que habría de erigirse como la ley suprema que fundamente la vida sin excepción (2018, pp. 41-46), "no es, como en las antiguas doctrinas, solo la prédica de una determinada virtud, sino la definición de la ley suprema de la vida humana y de la guía de conducta que deriva necesariamente de ella” (2018, p. 41). ¿Cómo funciona este mandato? Tolstoi sugiere la idea de un esfuerzo necesario para incrementar el amor en el interior del individuo (2018, p. 78), lo que provocaría un desarrollo de la conciencia, que aparece como una propiedad de la naturaleza humana $(2018$, p. 85$)$ que orienta la conducta hacia el amor a los demás. Una vez que la conciencia se vuelve más transparente y lúcida (2018, pp. 79-82), Tolstoi sostiene que se produce un salto social que provocaría que las sociedades no necesitaran ni siquiera un gobierno, sino solo la doctrina de Cristo (2018, p. 92). En definitiva, escribe el genio ruso, "Toda la doctrina radica en que aquello que llamamos «yo», nuestra vida, es un principio divino alojado en nosotros y limitado por nuestro cuerpo, principio que se manifiesta como amor; que, por tanto, la verdadera vida de cada hombre, divina y libre, se manifiesta en el amor" (2018, p. 42).

Y ahora, ¿qué principios pueden extraerse que sean comunes a la inmensa mayoría de estos autores?

\subsection{Los elementos básicos del amor cristiano}

A pesar de las diferencias desarrolladas anteriormente, existen unos principios que, por convergencia, concitarían el consenso de todos los autores.

El primer elemento es que el amor cristiano se fundamenta en la afirmación ontológica de que "Dios es amor" (1 Jn 4, 8) (Hildebrand, 1998, pp. 298-302; Thibon, 2010, p. 39; Balthasar, 2004, pp. 88-91, 101-102; Kierkegaard, 2006, p. 26; Lewis, 2017, pp. 18, 168-169, 180-184; Soloviev, 2009, pp. 52-53, 98, 101; Tolstoi, 2018 , pp. 28, 31, 78). Se comprendería la vida intratrinitaria 
como una dinámica relacional de amor entre las personas de la Trinidad, que afirmarían su propia identidad en relación con las otras, y la creación como el fruto del amor de Dios-Trinidad, que no se queda encerrado en esas relaciones previas, sino que tiende a darse de suyo. Ahora bien, el punto álgido del amor divino se sitúa en el grito del abandono y la muerte en la cruz. Todo lo cual ofrece una clave hermenéutica sustancial: el amor divino del que participa el ser humano a través del ágape, la caridad, no puede reducirse solo a una comprensión sacrificial, sino que ha de ampliarse a una relación de amor y de vida.

El segundo elemento tiene que ver con las tipologías del amor. Es cierto que hay distintos tipos de amor natural en función del objeto amado -y es así como podemos hablar de un amor paternal y maternal, fraternal, amistoso o esponsalicio-. Pero es unánime que el amor divino irriga, transfigura y sobrenaturaliza todos los tipos de amor natural al constituir su fundamento. Dios incoaría una parte de su amor en las personas, a su imagen y semejanza, y esto las capacitaría para que, con el concurso de su libertad, los diversos amores se fundamenten en ese primero y se desarrollen también hacia todos los demás. Así es posible hablar de un amor de los padres, de los hermanos, de los amigos, de los esposos que se nutre y se desarrolla por participación en el único amor divino (Hildebrand, 1998, pp. 305-306; Thibon, 2010, pp. 49, 86; Balthasar, 2004, pp. 77, 128-131; Kierkegaard, 2006, pp. 25, 133; Lewis, 2017, pp. 155 158, 176-177; Soloviev, 2009, pp. 95-98; Tolstoi, 2018, p. 79).

El tercer elemento es que el amor conduce a la unidad y se manifiesta en ella (Hildebrand, 1998, p. 173; Thibon, 2010, pp. 37-38, 46, 125, 137; Balthasar, 2004, p. 118; Kierkegaard, 2006, pp. 80, 136; Lewis, 2017, pp. 86-87, 174-176; Soloviev, 2009, pp. 52-53, 107, 111; Tolstoi, 2018, p. 104). Es preciso realizar dos matices aquí. Que la unidad generada por el amor es una unidad relacional recíproca y reciprocante. Es decir, que el amor pide de suyo ser amado, también el MN, aunque no con la persona que es objeto de amor en un momento determinado, sino en otras ocasiones y en función de la propia indigencia que necesita ser amada. Pero esta relación no puede quedar enclaustrada en la vinculación de unos padres con sus hijos, de los miembros de un matrimonio o unos amigos, sino que desborda hacia otras personas que se sienten llamados también a ese amor generando así un movimiento expansivo de nuevas relaciones. El segundo matiz es cognoscitivo. Porque este entramado de relaciones, fundamentadas en el amor divino, permitirían 
a las personas acceder a un conocimiento de la realidad que no es puramente gnóstico y que revela a Dios y el mundo creado por él también como fuente y parte de un equilibro de realidades relacionadas entre sí.

La dimensión moral del amor cristiano se mueve en torno a dos ideas. La primera, la obligación de amar a las personas (Hildebrand, 1998, pp. 288-302; Thibon, 2010, p. 39; Balthasar, 2004, pp. 112, 120; Kierkegaard, 2006, pp. 43 y ss.; Lewis, 2017, pp. 171-176; Soloviev, 2009, pp. 72-73; Tolstoi, 2018, pp. 41-46). En efecto, el MN no parece ser interpretado como una carga pesada, sino dentro de una lógica normativa que protege el amor divino frente a los ídolos, las inclinaciones naturales o la pura predilección. La segunda, la intuición de una conciencia moral irrigada por el amor divino que, de alguna forma, dirige el obrar humano de tal forma que exprese su amor (Hildebrand, 1998, pp. 314-319; Thibon, 2010, p. 39; Balthasar, 2004, pp. 60, 77, 106-107; Kierkegaard, 2006, pp. 169 y ss.; Lewis, 2017, p. 169; Soloviev, 2009, pp. 49-50, 110; Tolstoi, 2018, pp. 77-78). Se da por supuesto que los seres humanos tienen una conciencia moral, acaso como el lugar donde parece hacerse perceptible ese amor incoado, que actualiza el amor divino e intenta dirigir el obrar humano. Ambas dimensiones presuponen la necesidad de una ascesis -que no puede erigirse como un fin en sí mismo- que corrija esas tendencias contrarias al MN o a la conciencia moral.

Aunque cabría hablar de otros elementos básicos del amor cristiano, no concitan la unanimidad de los autores. Con todo, estos son suficientes para entablar un diálogo con los núcleos de discusión fundamentales en la pedagogía moderna, que es la segunda parte de este artículo.

\section{Segunda parte. diálogo abierto entre los elementos básicos DE UNA FILOSOFÍA CRISTIANA DEL AMOR Y LOS PRINCIPALES NÚCLEOS DE DISCUSIÓN ACTUALES SOBRE EL AMOR EN LA PEDAGOGÍA}

El análisis de la literatura existente revela tres núcleos de discusión fundamentales. Una serie de interpretaciones del amor en el ámbito educativo que casi se abren a un modo nuevo de comprender la educación epistemológicamente. Los otros dos núcleos forman parte de lo que se llama "triángulo peda- 
gógico" o los mitos sobre el origen de la docencia (McEwan, 2003) y serían la relación que mantienen los profesores y los estudiantes con el conocimiento, y la propia relación entre los profesores y los estudiantes.

\subsection{Pensar la educación a partir de nuevas propuestas del amor}

Como acabo de decir, parece haber un número de investigaciones que proponen nuevas formas de comprender el amor que casi plantean pensar la educación de otra manera. Podrían entrar en los apartados siguientes porque desarrollan argumentos muy próximos, pero tiene más sentido comprenderlos en este contexto. Aquí, inspirados por la figura paradigmática de Sócrates y el pensamiento de Hannah Arendt (Alston, 1991; Vlieghe y Zamojski, 2019, pp. 520-521), el profesor experimenta la necesidad de compartir su amor al mundo y, justo porque algo en él le emociona, lo transmite a sus estudiantes, que pueden recrearlo continuamente. Escriben los autores: "At an ontological level [...] educational love refers [...] to a particular attitude towards the world (affirmation), towards oneself (internal necessity) and towards others (vulnerability and generosity)" (Vlieghe y Zamojski, 2019, p. 523). Desde perspectivas más pragmatistas, se produce una identificación entre la noción de "amor evolutivo" que desarrolla Charles Peirce y su mismo concepto de educación, donde el proceso educativo se identificaría con un proceso de evolución de la sociedad en la atención a los problemas sociales y los más desfavorecidos (Moses, 2017, pp. 724-725). La pedagogía crítica aprovecha el uso ambiguo del amor que aparece en Paulo Freire para articular una idea de "amor revolucionario" que intentaría romper la lógica de la indignación y la ira en que se mueve la vida política actual, volviéndose más sensible a las necesidades locales concretas y ofreciendo prácticas de amor que permitan cambiar esas mismas situaciones en una apertura radical al otro (Lanas y Zembylas, 2014, pp. 40-42; Zembylas, 2017, pp. 5-8, 10-11). Hoveid y Finne proponen repensar la educación introduciendo, en el ámbito de las relaciones educativas de cuidado, los atributos que ellos llaman "in between", que sería como el espacio entre dos personas que define el tipo de relación que se desarrollará, y “open doors", que constituye la actitud de mantenerse siempre abierto a los demás (Hoveid y Finne, 2014, pp. 248-25, 253-257). 
Qué decir desde una filosofía cristiana del amor que no pronuncia palabra alguna sobre la educación.

Si hubiera que pensar qué es la educación en términos estrictamente pedagógicos y partiendo de las bases que he deducido del primer bloque, sería necesario partir de la vinculación entre Dios y el ser humano en virtud del concepto de amor. Se observaba que los seres humanos tienen dentro de sí un germen del amor divino cuyo despliegue permitía reconocer la verdad de la revelación de Dios -que se comunicaba trinitariamente como amor-, responder a la revelación a través de nuestra libertad -que se hacía amor en la obediencia ascética al MN y a la conciencia moral- y conformaba así una identidad particular en la manifestación idiosincrática del amor divino a través de los amores naturales. Como decía -y aun sabiendo que lo que voy a decir es reduccionista-, en virtud de estos argumentos cabría pensar la educación como una actualización progresiva del amor de Dios incoado en el ser humano que se manifestaría en la dimensión intelectual, moral, política y espiritual del ser humano. Así se puede hablar de una forma de conocer la realidad como inmersa en las lógicas del amor divino que exige la iniciación en los diversos lenguajes del conocimiento humano y un pensamiento crítico capaz de comprender la realidad bajo esas dinámicas. Se puede hablar de una moral que se desarrolla en el hábito de amar a través de experiencias concretas que brotan de la obediencia al MN y que aprende a discernir la voz de la conciencia moral entre todas las otras voces. Se puede hablar de una política como forma de vida que se desarrolla en el pensamiento crítico, el diálogo y la vida práctica. Y se puede hablar de una espiritualidad que se condensa en las relaciones recíprocas y reciprocantes que establecen los seres humanos con el entorno que les rodea. Nada de esto es incompatible con que pueda existir un sistema específico de formación catequética, cuyo objeto de estudio pertenece más al lenguaje de la teología práctica que al de la teoría educativa. Tampoco es incompatible con la formación específica que cada persona de fe puede adquirir a través de los medios que las diversas religiones articulan -porque no vienen sino a dar una forma específica a ese amor se manifiesta que, idiosincráticamente, en la persona concreta vive un carisma particular-. Por supuesto, no es incompatible con otras realidades eclesiológicas como los sacramentos, que constituyen manifestaciones del amor de Dios hacia el ser humano y contribuyen también al desarrollo de ese amor divino incoado que hace a cada hombre y a cada 
mujer a imagen y semejanza de Dios-Trinidad. En definitiva, la educación que acontece en los sistemas formales de educación -la que se propone repensar aquí- participa de todo este entramado a partir de sus atributos específicos.

\subsection{La relación de los profesores y los estudiantes con el conocimiento. El amor a la asignatura entre la enseñanza y el aprendizaje}

A pesar de que vivir este amor al conocimiento no parece ser una tarea facilitada por las políticas educativas internacionales (Gary, 2019, pp. 479483; Taubman, 2017; Ball, 2003) e, incluso, induce a algunos docentes a una cierta desesperación (Liston, 2000, pp. 81-89; Ball, 2003), se puede hablar de cinco grandes argumentarios. El primero apareció sugerido anteriormente: se entiende que los profesores aman una parte del mundo que transmiten en sus asignaturas permitiendo, así, que cada nueva generación la conozca y pueda transformarla con la misma pasión que sus profesores tienen al enseñar lo que aman (Assiter, 2013; Koorsgaard, 2019, pp. 12-13). Esa misma pasión constituye el segundo núcleo: "Passion brings a teacher's subject matter of life. A teacher's passion is infectious and easily engenders the student's interest. When a teacher's passion for his subject matter is genuine and committed, it shows itself and transforms students; they too become believers in this importance" (Anderson, 2002, p. 45). El tercer punto es que el amor al conocimiento exige una razón diferente. Elliot propone una escala que va del amor a la educación en sí misma, al amor contemplativo de la parte del mundo que representa esa asignatura hasta alcanzar un amor al mundo entero que se refleja en la unidad y la relación de los contenidos curriculares (Elliot, 1974). Rocha propone una razón erótica que se produce en el marco de intuiciones que permiten la inclusión de la sensibilidad en el proceso cognitivo (Rocha, 2009, p. 586). El cuarto núcleo es una interpretación platónica del amor al conocimiento. Platón parecía sugerir que el conocimiento desarrolla nuestras almas y se enriquece cuando aprendemos junto a quienes admiramos y amamos hasta el punto de que se produce un salto que incurre en moralidad, pues la verdad es performativa (Hinchliffe, 2006). Esto lleva al último punto: las posibilidades de un conocimiento moral. En la educación del carácter sería necesario lograr que los estudiantes amen la virtud, para lo que se necesita que sea significativa, se 
adapte a la propia persona y le facilite experiencias que la hagan feliz. En este sentido, el amor se erige como un elemento en la definición de la identidad a través de los "habits of being", pequeñas experiencias concretas de amor que terminan definiendo la propia identidad más que un conjunto de virtudes ya prescritas de antemano (Graham, 2018).

¿Qué pude decir una filosofía cristiana del amor respecto de estas ideas?

La filosofía cristiana del amor propondría un primer amor a Dios del que nacería todo amor posible en educación. No obstante, en la línea de las intuiciones de Elliot, llevaría a discernir la huella creadora de Dios-Amor en la parte del mundo que se ama a través de la propia asignatura como una parte relacionada con todas las demás. Esta intuición afecta sustancialmente al diseño del currículo, que habría de pensarse en una estructura donde las áreas de conocimiento evidencien la relación interna que mantienen entre sí. Esta idea exige matizar algunos aspectos sobre la pasión del profesorado que parece ser tan común en la bibliografía. El argumento sobre el que reposa esta idea debe englobarse dentro de una pasión más omnicomprensiva por la propia educación dentro de la cual, ahí sí, ha de privilegiarse el amor a la educación -que no aparece en ningún artículo de los estudiados, por cierto-.

Así, la propia asignatura debería poner en relación el mundo que representa y los estudiantes de un aula en concreto. Uno de los fines de todos los profesores en cualquier asignatura sería lograr que los estudiantes adquieran el hábito de interpretar la realidad desde el horizonte del amor de Dios-Trinidad, esto es, en las múltiples relaciones que mantienen todas las dimensiones de la realidad. Con todo, es necesario puntualizar tres cosas. Que a pesar de las buenas intenciones que cabría escribir aquí, la esencia última de la realidad permanece en el misterio. Que esta pretensión no se logra únicamente con una enseñanza directiva, sino con métodos que desafían los aparentes límites que se dan en la educación formal. Y que el conocimiento que se puede lograr no es puramente cognitivo, sino que nace de las relaciones recíprocas y reciprocantes de un aula. Lo que nos conduce a la dimensión moral del conocimiento.

Porque es necesario aprender a amar. Esta idea se divide en las implicaciones del MN y la conciencia moral que, en realidad, son como dos vías aproximativas e interrelacionadas a una única realidad. El aprendizaje y la interiorización del MN, que es la norma de cualquier norma que se pueda proponer para regular la vida del aula -especialmente, en las edades más tempranas-, comienzan a 
dar una orientación moral a los actos que ya sugiere la conciencia de una forma tenue y que se intensifican a medida que el amor crece dentro de la persona al estar en contacto con personas que ama. Los profesores deben entender que estas tendencias deben educar en el silencio y el diálogo en lo que tiene que ver con la conciencia moral tanto en los "habits of being" que, a fuerza de realizar experiencias concretas de amor y reflexionar sobre ellas e incluso comunicarlas, refuerzan la identidad de los estudiantes.

\subsection{La relación entre profesores y estudiantes}

Esta perspectiva busca interpretar la relación educativa en función de otros tipos de amor. Más allá de las propuestas que hablan del amor de una madre (Klein, 1989), de un amor inspirado en el romanticismo británico (Halpin, 2006) o de una reparación moral que reconocería a los grupos más débiles (Frank, 2011, pp. 598-600), la literatura oscila entre los presupuestos del eros y del ágape. El eros, que posee el mayor número de publicaciones, sostiene que los profesores y los estudiantes experimentan atracción los unos sobre los otros -no está exenta de ambigüedades y de posibles connotaciones sexuales (Todd, 2003; Hogan, 1993)-. En sus versiones más extremas e indefendibles, como la teoría del liking, se plantea gustar y seducir como el elemento nuclear de la formación hasta el punto de disociar el cuerpo del amor para reducirlo solo al físico (Burke y Greteman, 2013). ¿Pero qué hay en las propuestas más moderadas? Ahí, la pretensión fundamental del eros es engendrar, quiero decir, transformar a los estudiantes para que abran nuevas posibilidades en la realidad y llevarlas a efecto a través de lo aprendido -y que no simplemente imiten a sus profesores o estos quieran que reproduzcan lo que ellos (Kenklies, 2019)-. Pero esa atracción que ejercen los profesores en el ejercicio de su magisterio es solo un instrumento, ya digo que ambiguo y frágil, puesto al servicio de la educación y que exige que el docente aprenda a desviarla hacia la asignatura (Schwab, 1954; Aldridge, 2019), lo que implica conocer los límites de su tarea y saber que el cuidado tiene primacía sobre el eros (Williams, 2019). El ágape no ha llamado mucho la atención de los académicos y, cuando lo ha hecho -como dije en la introducción-, aparece en una interpretación casi siempre sacrificial que oscila entre San Agustín y Santo Tomás. Desde el punto de vista 
puramente relacional, advierte sobre la responsabilidad de los profesores sobre los propios estudiantes - lo que no significa necesariamente que deba darse una apertura emocional (Anderson, 2002)-. En términos agustinianos, donde si se ama a Dios no hay lugar para conducta desordenada alguna, es la liturgia la que revela la pretensión pedagógica más alta porque permite traer al presente la realidad escatológica de cada ser humano (Sosler, 2018), lo que tiene que ver también con una comprensión litúrgica del eros (Renga, 2017, pp. 268-273). En términos morales todavía más estrictos, es muy interesante la vinculación que se da entre phronesis y ágape en algunas relecturas tomistas, donde el amor aparece como el criterio de discernimiento que usa la prudencia para moverse entre el conocimiento de la realidad universal y particular y las condiciones y la finalidad del propio acto moral (Wivestad, 2008; 2012).

¿Qué decir desde los elementos básicos de una filosofía cristiana del amor?

Hay que comenzar como en el punto anterior: diciendo que hay un primer amor a Dios del que nacería todo amor posible en educación -también el que se da interpersonalmente-. A esta hay que añadirle, además, que la filosofía cristiana del amor no podría reducir la relación educativa a la relación exclusiva entre un profesor y un estudiante en virtud de la idea de relaciones recíprocas y reciprocantes. En este sentido, el amor cristiano que habría de irrigar las relaciones educativas amplía su perspectiva a todos los otros agentes educativos que no están en el aula concreta donde un profesor o un estudiante dan clase: los profesores y estudiantes de otras clases, las personas de administración y servicios, las familias. Así, el amor que da forma a la relación entre profesores y estudiantes se amplía a todos los actores de una institución educativa. Más todavía: cabría considerar la necesidad de que esas relaciones ascendieran al nivel institucional y se expandieran a los distintos centros de un mismo distrito educativo. La pregunta ahora es: ¿qué amor es el que preside esas relaciones?

Como he dicho, pueden existir formas de amor natural que ayudan a leer la relación educativa, como el eros, la philia o el cuidado, y que no son ajenas al amor divino. Pero es necesario precisar dos cosas a este respecto. Que cualquiera de esos amores está fundamentado en el amor de Dios, que sería el que revelaría sus límites y sus posibilidades formativas. Y que junto con ellos es necesario hablar de una relación educativa con sentido propio, casi como otra forma de amor natural, que es irrigada también por el amor divino y que abre los argumentos a términos mucho más pedagógicos que todos los 
anteriores. Ahí, los profesores y los estudiantes se congregan en un aula, a la luz de un proceso de enseñanza y aprendizaje inspirado por distintas teorías y bajo diversas metodologías, para conocer la realidad y aprender a amar moralmente en los términos que describí con anterioridad. Fundamentado en el amor de Dios, eso sí, esa relación educativa revelaría un conocimiento de la realidad alternativo al puramente cognitivo y una orientación moral inspirada y dirigida hacia el amor.

\section{Conclusiones}

A la vista de la pequeña historia sobre la filosofía cristiana del amor que se desarrolló en el siglo xx, es posible abstraer una serie de principios que arrojan una visión del amor cristiano renovada, que habría de abrirse a otras obras que no he podido tratar aquí por razones de espacio, como la de Maurice Nédoncelle, a otras lógicas de pensamiento, como la renovación de los estudios trinitarios que ha concitado a Rahner, Greshake, Florensky o Hemmerle, a la mística, donde cabría hablar de Adrienne Von Speyr o Chiara Lubich, o a otras religiones, donde aparecerían Erich Fromm, Etty Hillesum y George Steiner. Esos principios sería que cualquier amor del que quepa hablar en educación ha de fundamentarse en Dios-Amor, que habría incoado en cada persona un amor a su imagen y semejanza. Este amor haría posible discernir la realidad como un entramado de relaciones recíprocas y reciprocantes y adoptaría la forma de la libertad humana, transformando cada amor natural y expresándose moralmente en el MN y la conciencia moral.

Sentados estos principios, se puede establecer un diálogo con las discusiones pedagógicas actuales sobre el amor donde se puede intuir lo siguiente.

Que se podría comprender el fenómeno educativo como una actualización del germen del amor divino que hay en cada ser humano. Así, cualquier amor posible que tenga lugar en la educación se sustenta sobre Dios-Amor. El amor al conocimiento habría de plasmarse en un currículo donde cada lenguaje de conocimiento expresara sus relaciones internas con todos los demás para hacer posible que los estudiantes puedan comenzar a ver la realidad así. A ello se llega no solo cognitivamente, en una iniciación en los diversos lenguajes del conocimiento, sino como fruto de las relaciones recíprocas y reciprocantes 
que mantienen los distintos agentes de la comunidad educativa. Ahí, aparece la vida moral de las aulas en el marco del amor cristiano, en el cumplimiento del MN y a la escucha de la conciencia moral, que se expresan en pequeños actos de amor concretos que conforman la identidad de los estudiantes.

\section{BiBLIOGRAFÍA}

Aldridge, D. (2019). Education's Love Triangle. Journal of Philosophy of Education 53(3), 531-546. DOI: https://doi.org/10.1111/1467-9752.12373 Alston, K. (1991). Teaching, Philosophy, and Eros: Love as a Relation to Truth. Educational Theory 41(4), 385-395. DOI: https://doi.org/10.1111/j.17415446.1991.00385.X

Anderson, D. R. (2002). Creative Teachers: Risk, Responsibility, and Love. Journal of Education 183(1), 33-48. DOI: https://doi. org/10.1177/002205740218300104

Assiter, A. (2013). Love, Socrates, and Pedagogy. Educational Theory 63(3), 253-263. DOI: https://doi.org/10.1111/edth.12022

BALL, S. J. (2003). The teacher's soul and the terrors of performativity. Journal of Education Policy 18(2), 215-228. DOI: https://doi. org/10.1080/0268093022000043065

Burke, K. y Greteman, A. (2013). Toward a Theory of Liking. Educational Theory 63(2), 151-170. DOI: https://doi.org/10.1111/edth.12015

Elliott, R. K. (1974). Education, Love of One's Subject, and the Love of Truth. Journal of Philosophy of Education 8(1), 135-153. DOI: https://doi. org/10.1111/j.1467-9752.1974.tb00493.x

FrANK, J. (2011). Love and ruin(s): Robert Frost on moral repair. Educational Theory 61(5), 587-600. DOI: https://doi.org/10.1111/j.17415446.2011.00422.x

Gary, K. (2019). Pragmatic Standards versus Saturated Phenomenon: Cultivating a Love of Learning. Journal of Philosophy of Education 53(3), 477-490. DOI: https://doi.org/10.1111/1467-9752.12377

Graham, P. (2018). Lost and Found: Personal Reflections on Educational Earnestness and the Power of Love. Educational Theory 68(2), 161-176. DOI: https://doi.org/10.1111/edth.12304 
Halpin, D. (2006). Why a Romantic Conception of Education Matters. Oxford Educational Review 32(3), 325-345.

Hinchliffe, G. (2006). Plato and the love of learning. Ethics and Education 1(2), 117-131.

Hogan, P. (1993). The Practice of Education and the Courtship of Youthful Sensibility. Journal of Philosophy of Education 27(1), 5-17. DOI: https:// doi.org/10.1111/j.1467-9752.1993.tb00292.x

Hoveid, M. H. y Finne, A. (2014). "You Have to Give of Yourself”: Care and Love in Pedagogical Relations. Journal of Philosophy of Education 48(2), 246-259. DOI: https://doi.org/10.1111/1467-9752.12069

Kenklies, K. (2019). The Struggle to Love. Pedagogical Eros and the Gift of Transformation. Journal of Philosophy of Education 53(3), 547-559. DOI: https://doi.org/10.1111/1467-9752.12376

KierKegaARD, S. (2006). Las obras del amor. Salamanca: Sígueme.

KleIn, J. T. (1989). Teaching and Mother Love. Educational Theory 39(4), 373-383. DOI: https://doi.org/10.1111/j.1741-5446.1989.00373.x

Lanas, M. y Zembylas, M. (2014). Towards a Transformational Political Concept of Love in Critical Education. Studies in Philosophy and Education 34(1), 31-44. DOI: https://doi.org/10.1007/s11217-014-9424-5

Lewis, C. S. (2017). Los cuatro amores. Madrid: Rialp.

Liston, D. P. (2000). Love and despair in teaching. Educational Theory 50(1), 81-102. DOI: https://doi.org/10.1111/j.1741-5446.2000.00081.x

McEwan, H. (2003). The primitive artist and the lover: two stories of the origins of teaching. Educational Theory 53(4), 421-436. DOI: https://doi. org/10.1111/j.1741-5446.2003.00421.x

Moses, R. G. (2017). Groundworks for a Pedagogy of Evolutionary Love Ethics: Archetypes of Moral Imagination in the Pragmatisms of Peirce and Addams. Educational Theory 67(6), 713-725. DOI: https://doi.org/10.1111/ edth. 12282

RenGA, I. P. (2017). Unpacking a Liturgical Framing of Desire for the Purposes of Educational Research. Educational Studies 53(3), 263-284. DOI: https:// doi.org/10.1080/00131946.2017.1303495

Rocha, S. (2009). A return to love in William James and Jean-Luc Marion. Educational Theory 59(5), 579-588. DOI: https://doi.org/10.1111/j.17415446.2009.00339.x 
SchwAB, J. (1954). Eros and education: a discussion of one aspect of discussion. The Journal of General Education 8(1), 51-71.

Soloviev, V. (2009). El significado del amor. Burgos: Monte Carmelo.

SosLer, A. (2018). Reason, Love, and Morality: the Limits of Reason in Kohlberg and the Importance of Love in Augustine and Smith. Religious Education, 1-13. DOI: https://doi.org/10.1080/00344087.2018.1492290

Taubman, P. (2017). Death by Numbers: A Response to Backer, Sarigianides, and Stillwaggon. Educational Theory 67(1), 97-106. DOI: https://doi. org/10.1111/edth.12230

ThiBon, G. (2010). Sobre el amor humano. Madrid: El buey mudo.

Todd, S. (2003). A Fine Risk To Be Run? The Ambiguity of Eros and Teacher Responsibility. Studies in Philosophy and Education 22(1), 31-44. DOI: https://doi.org/10.1023/A:1021133410527

Tolstói, L. (2018). Ley de la violencia y ley del amor. Madrid: Hermida Editores.

Vlieghe, J. y Zamojski, P. (2019). Out of Love for Some-Thing: An Ontological Exploration of the Roots of Teaching with Arendt, Badiou and Scheler. Journal of Philosophy of Education 53(3), 518-530. DOI: https://doi. org/10.1111/1467-9752.12375

Von Balthasar, H. U. (2011). Sólo el amor es digno de fe. Salamanca: Sígueme.

Von Hildebrand, D. (1998). La esencia del amor. Navarra: EUnSA.

Williams, K. (2019). "How Can We Know the Dancer from the Dance?" Personal Concern and Sexual Desire in the Educational Relationship. Journal of Philosophy of Education 53(3), 560-573. DOI: https://doi.org/10.1111/14679752.12378

Wivestad, S. M. (2008). The Educational Challenges of Agape and Phronesis. Journal of Philosophy of Education 42(2), 307-324. DOI: https://doi. org/10.1111/j.1467-9752.2008.00626.x

Wivestad, S. M. (2012). On Becoming Better Human Beings: Six Stories to Live By. Studies in Philosophy and Education 32(1), 55-71. DOI: https:// doi.org/10.1007/s11217-012-9321-8

ZEMBYLAS, M. (2017). Love as ethico-political practice: Inventing reparative pedagogies of aimance in "disjointed" times. Journal of Curriculum and Pedagogy 14(1), 23-38. DOI: https://doi.org/10.1080/15505170.2016.127 7572 